\title{
Schlafstörungen und COVID-19-Erkrankung
}

Häufig werden Schlafstörungen durch Stress ausgelöst. Ausnahmesituationen wie die COVID-19-Erkrankung können unterschiedliche Gefühle wie z. B. Besorgnis, Verunsicherung oder Angst auslösen und Patientlnnen in einen Stresszustand versetzen.

Ein Jahr nach Beginn der Pandemie und zum Weltschlaftag am 19. März zog Schlafforscherin Dr. Anna Heidbreder vom Schlaflabor der Innsbrucker Universitätsklinik für Neurologie Bilanz.

Zu Beginn der Pandemie haben Daten - vorwiegend aus China - gezeigt, dass es infolge einer COVID-19-Erkrankung wesentlich häufiger zu Schlafstörungen kommen kann. Haben sich die Zahlen nach einem Jahr bestätigt?
Diese Zahlen haben sich mittlerweile vielfach bestätigt. So wurde aus der ganzen Welt eine Reihe von Arbeiten publiziert, die zeigen, dass es während und in Folge einer COVID-19-Erkrankung zu Schlafstörungen kommen kann. Auch an unserer Ambulanz melden sich immer noch Patientinnen und Patienten, die in Folge einer COVIDErkrankung schlaflos geworden sind.

Was berichten die Patienten?
Die Insomnie zeigt sich vorwiegend als Einschlafstörung, zu frühes Aufwachen oder als nicht erholsamer Schlaf. Bei Patienten, die eine COVID-19-Erkrankung durchgemacht haben und mittlerweile schon wieder arbeiten, führt dies oft zu einer erheblichen Beeinträchtigung, da sie sich Sorgen machen, ob sie die benötigte Leistung erbringen können, wenn sie nachts nicht richtig schlafen.

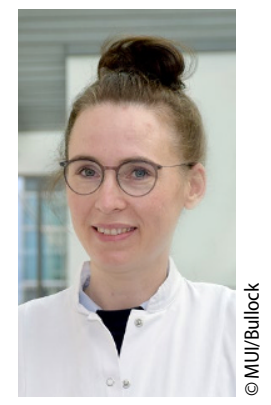

Dr. Anna Heidbreder Universitätsklinik für Neurologie Innsbruck

Hier steht eine Anzeige. 


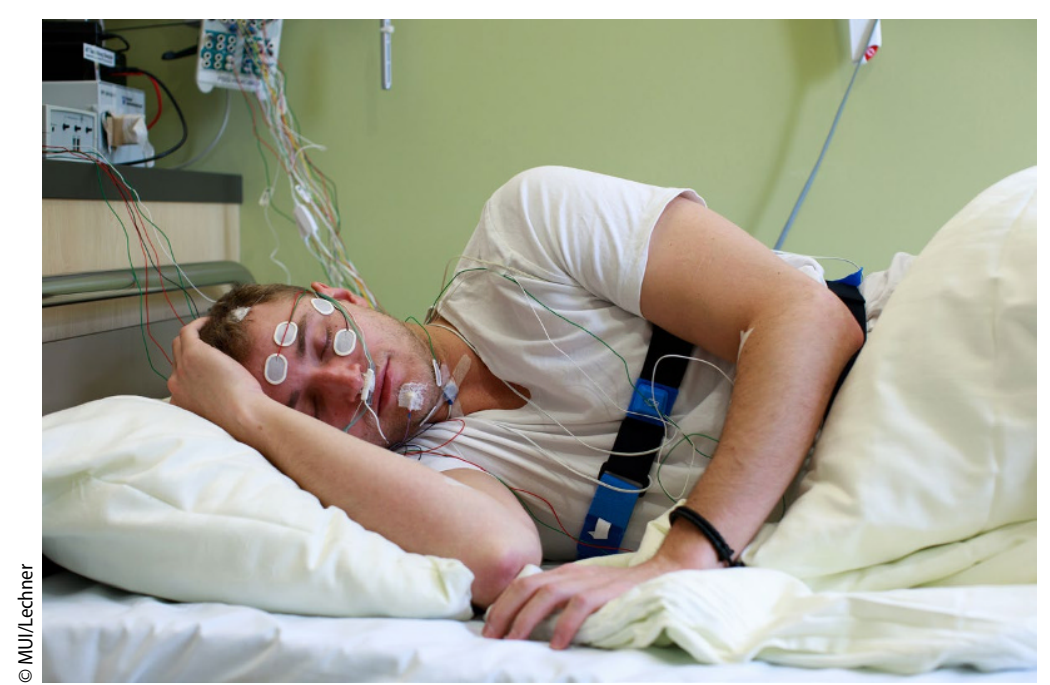

$\Delta$ Im Schlaflabor zeigte sich unerwarteter Weise, dass ein Teil der Patienten Auffälligkeiten im REM-Schlaf aufwies

Kann die Ursache aus diesen Berichten auf das neuartige CoronaVirus zurückgeführt werden?

Die Ursachen sind vielfältig. Eine ganze Reihe kann davon jedoch mit SARS-Cov-2 in Zusammenhang gebracht werden. So würde z.B. eine vermehrte Stressbelastung zu einem Zustand von vermehrten Arousals, zu intrinsischen Mikro-Weckreaktionen führen. Für viele Betroffene ist die COVID-19-Erkrankung eine sehr traumatische Erfahrung. Dies betrifft natürlich Patientinnen und Patienten, die auf einer Intensivstation gelegen haben, aber auch andere mit vermeintlich minder schweren Verläufen. Bei manchen Patientinnen und Patienten sind die Schlafstörungen somit als TraumaFolge zu sehen. Hier kommen zur Schlaflosigkeit oft auch Albträume hinzu. Aber auch sonst sind die psychischen Folgen oft sehr ausgeprägt, und mittlerweile gibt es eine ganze Reihe von Untersuchungen, die das bestätigt haben - auch aus Innsbruck und anderen Teilen Österreichs.

k: https://www. psychosomatik-innsbruck.at

Quelle: Medizinische Universität Innsbruck
Unsere Gruppe in der Neurologie konnte eine kleine Anzahl von Patienten im Schlaflabor untersuchen, die an der Univ.-Klinik für Innere Medizin II betreut werden. Ein unerwarteter Befund war hier, dass ein Teil eine Auffälligkeit im REM-Schlaf aufgewiesen hat; d.h. im REM-Schlaf war bei diesen Patienten der Muskeltonus nicht aufgehoben (wie es normalerweise der Fall ist), sondern es war eine vermehrte muskuläre Aktivität vorhanden. Dies könnte ein möglicher Hinweis darauf sein, dass die SARS-Cov-2-Erkrankung bei diesen tatsächlich das zentrale Nervensystem mit betrifft. Ob dies auf Dauer so bleibt, kann man naturgemäß noch nicht sagen, da Langzeitbeobachtungen fehlen. Außerdem ist nicht nur die COVID-19-Erkrankung an sich, sondern auch die mit der Erkrankung und Pandemie verbundenen oft existenzbedrohenden beruflichen und wirtschaftlichen Konsequenzen ein ganz wesentlicher Mitgrund für die Schlafstörungen. Neue wissenschaftliche Untersuchungen aus Singapur zeigen, dass es durch die COVID-19-bedingte Mobilitätsrestriktion zu Auswirkungen auf Schlaf und zirkadiane Rhythmen kommt - das gleiche gilt für verminderte Tageslichtexposition.

Was kann man gegen solche Schlafstörungen tun?

Es gibt eine Reihe von neuen und hilfreichen Interventionen. So hat beispielsweise das Department für Psychiatrie, Psychotherapie und Psychosomatik ein Online-Interventionsprogramm für Betroffene gestartet. Es gibt jedoch auch eine andere Seite: Mittlerweile gibt es eine Reihe von Patienten, die durch die COVID-19-bedingten Einschränkungen der Bewegungsfreiheit und Homeoffice mehr Zeit zum Schlafen hatten. Eine Umfrage von Brigitte Holzinger vom Wiener Institut für Bewusstseins- und Traumforschung und ihrem Team hat genau dies gezeigt: Manche Patienten konnten endlich besser ausschlafen. Auch in Argentinien haben Schlafmediziner bereits zu Anfang der Pandemie ihre Patienten dazu aufgerufen, den Lockdown zu nützen und das oft ausgeprägte chronische Schlafdefizit auszugleichen, was bei einem Teil auch gelungen ist.

Wie lange dauert es, bis es wieder zu normalen Schlafgewohnheiten kommt?

Da die Pandemie gerade erst einmal ein Jahr alt ist, fehlen Langzeitbeobachtungen.

Hinweis des Verlags. Der Verlag bleibt in Hinblick auf geografische Zuordnungen und Gebietsbezeichnungen in veröffentlichten Karten und Institutsadressen neutral.

psychopraxis.neuropraxis2021·24:153-155 https://doi.org/10.1007/s00739-02100730-3

() Springer-Verlag GmbH Austria, ein Teil von Springer Nature 2021 
Hier steht eine Anzeige.

Springer 